\title{
Study on the interaction between ketoprofen and bovine serum albumin by molecular simulation and spectroscopic methods
}

\author{
Jin Lian Zhu ${ }^{\text {a }}$, Jia He ${ }^{\text {a }}$, Hua He ${ }^{\text {a,b,c,*, Shu Hua Tan }}{ }^{\text {d }}$, Xiao Mei He ${ }^{\text {a }}$, Chuong Pham-Huy ${ }^{\text {e }}$ and \\ Lun $\mathrm{Li}^{\text {a }}$ \\ ${ }^{a}$ China Pharmaceutical University, Nanjing, China \\ ${ }^{\mathrm{b}}$ State Key Laboratory of Natural Medicines, China Pharmaceutical University, Nanjing, China \\ ${ }^{\mathrm{c}}$ Key Laboratory of Drug Quality Control and Pharmacovigilance, China Pharmaceutical University, \\ Ministry of Education, Nanjing, China \\ ${ }^{\mathrm{d}}$ School of Life Science and Technology, China Pharmaceutical University, Nanjing, China \\ ${ }^{\mathrm{e}}$ Faculty of Pharmacy, University of Paris V, Paris, France
}

\begin{abstract}
The interaction between ketoprofen and bovine serum albumin (BSA) was investigated by molecular simulation, fluorescence and UV-vis spectroscopy methods under the simulated physiological conditions. Molecular simulation method performed to reveal the possible binding mode or mechanism suggested the binding forces between ketoprofen and BSA were mainly hydrophobic interaction and hydrogen bond, which was in agreement with the thermodynamic study $\left(\Delta H^{\Phi}\right.$ and $\Delta S^{\Phi}$ were calculated to be $74.514 \mathrm{~kJ} / \mathrm{mol}$ and $333.98 \mathrm{~J} / \mathrm{mol} \cdot \mathrm{K}$ ). The binding constants of ketoprofen and BSA at different temperatures $(298,310$ and $318 \mathrm{~K})$ were calculated according to the data obtained from fluorescence spectra and the results indicated that ketoprofen had strong ability to quench the intrinsic fluorescence of BSA via a combination of static and dynamic quenching. Meanwhile, the changes of the conformation of BSA caused by ketoprofen were qualitatively analyzed with the UVvis and synchronous fluorescence spectroscopy. The distance between ketoprofen and tryptophan residue in BSA was calculated to be $1.58 \mathrm{~nm}$.
\end{abstract}

Keywords: BSA, ketoprofen, molecular simulation, fluorescence spectra, UV-vis spectra

\section{Introduction}

Recently, studies with protein have received much interest in life sciences, chemistry, and medicine as they showed broad and promising applications in the area of rational drug design $[11,14]$ and facilitated to understand binding mechanism of small molecule drugs and protein. Serum albumin, the most abundant protein in plasma, can be combined with many endogenous and exogenous ligands playing an important role in storage and transport of energy [22,27]. Bovine serum albumin, one of the widely used serum albumins owing to its cheapness and availability, specific ligand binding [9], is often used as a model protein instead of human serum albumin to investigate the mechanism of small molecules and protein.

\footnotetext{
${ }^{*}$ Corresponding author: Hua He, China Pharmaceutical University, Nanjing 210009, China. Tel./Fax: +86 02583271505 ; E-mail: dochehua@163.com.
} 
<smiles>CC(C(=O)O)c1cccc(C(=O)c2ccccc2)c1</smiles>

Fig. 1. Structure of ketoprofen.

Ketoprofen (Fig. 1), 2-(3-benzoylphenyl)-propionic acid, an arylpropionic acid derivative, is one of the most useful aryl alkyl acid non-steroidal anti-inflammatory drugs (NSAIDs). Its anti-inflammatory effect is approximately 160 times of aspirin on a unit weight basis [15] and its pharmacological activity is primarily on the inhibition of cyclooxygenase (also known as cyclooxygenase, COX) created during the metabolism of arachidonic acid and decreases the synthesis of prostaglandins (PGs), thereby acting as a antipyretic, analgesic and anti-inflammatory agent to moderate various pain such as postoperative pain, dental pain, acute visceral pain, acute muscle injury and pain, chronic cancer pain and primary dysmenorrheal. In clinical, it is also used as an anti-rheumatic drug, commonly used to treat ankylosing spondylitis, rheumatoid arthritis and osteoarthritis.

Spectroscopy because of its high sensitivity, rapidity and ease of implementation, has been widely used to investigate drug binding with serum albumins [12,24]. However, problems such as binding sites of small drug molecules with serum albumins and effects of drugs on conformational changes of serum albumins have not been solved. Recently, molecular simulation method is employed to analyze, investigate, and predict ligand-protein interactions as it can rationalize the experimental results and sometimes provide essential missing pieces of information which is not obtained by other means [19]. However, a major drawback of current molecular docking methods is the oversimplification of the binding process (e.g., rigid proteins, single conformation of ligands), which results in low predictive accuracy of the scoring functions stems from these computationally convenient assumptions [10]. Thus, the combination molecular simulation method with spectroscopy redeemed pitfalls mutually. Some investigations on interaction between small drug molecules and proteins by molecular simulation and spectroscopy methods have been reported $[4,13,23]$, investigation of bonding mechanism by molecular simulation method is not mature and in many articles, the crystallographic structure of human serum albumin is often used to replace the crystallographic structure of bovine serum albumin, few gave the crystallographic structure of bovine serum albumin. In this work, the interaction between ketoprofen and BSA was investigated by means of molecular simulation as well as fluorescence, UV-vis spectroscopy under the simulated physiological conditions. The molecular docking method was used to study the mode of action between BSA and ketoprofen; the binding constants $K$ and thermodynamic data were obtained at different temperatures by fluorescence spectroscopy; the main binding force was speculated and the distance between BSA and ketoprofen was calculated according to the energy transfer mechanism, which provided referenced experimental data and theoretical basis for discussing the mechanism of NSAID drugs.

\section{Experiments}

\subsection{Apparatus and reagents}

A fluorescence spectrophotometer (RF5301, Shimadzu Company, Japan) was used for recording the fluorescence spectra and measuring the intensity of fluorescence. A UV-vis spectrophotometer (UV1800, Shimadzu Company, Japan) was used for recording the absorption spectra. A pH meter (pHS-25, Shanghai Wei Ye Instrument Company, Ltd, China) was used for $\mathrm{pH}$ measurement. 
Bovine serum albumin (BSA) was purchased from Nanjing Da Zhi Biological Technology Company (China) and its stock solution was prepared with $\mathrm{pH} 7.40,0.05 \mathrm{~mol} / \mathrm{l}$ Tris-HCl buffered salt solution (containing a concentration of $\mathrm{NaCl} 100 \mathrm{mmol} / \mathrm{l}$ ) to a concentration of $5 \mu \mathrm{mol} / \mathrm{l}$ and stored at $4^{\circ} \mathrm{C}$ in the darkness. Ketoprofen was purchased from Hubei Xun Da Pharmaceutical Co. Ltd, and its stock solution ( $3 \mathrm{mmol} / \mathrm{l}$ ) was prepared by $75 \%$ methanol; Tris (Tris- $\mathrm{HCl}$ ) buffered solution was prepared by mixing $2.4228 \mathrm{~g}$ of Tris and $1.1700 \mathrm{~g}$ of $\mathrm{NaCl}$ in $200 \mathrm{ml}$ distilled water, and then $\mathrm{pH}$ of the solution was adjusted with $0.1 \mathrm{~mol} / \mathrm{l}$ hydrochloric acid to 7.40 ; the water used to prepare solution was double distilled water; other reagents were of analytical reagent grade and above.

\subsection{Experimental method}

Molecular simulation: Considering the crystallographic structure of BSA was not included in the Protein Data Base (PDB), the three-dimensional feature of its model was obtained by a homology modeling procedure on the basis of the crystal structure of HSA, which was selected from the complexation of HSA and hemoglobin in PDB (PDB code: 1n5u) [25]. Homology modeling is an efficient method for the three-dimensional structure construction, besides, an optimal sequence alignment is essential to the success of homology modeling [25].

The building of protein models by homology modeling procedure usually proceeds along a series of well-defined and commonly accepted steps: (1) sequence alignment between the target and the template; (2) building an initial model; (3) refining the model; (4) evaluating the quality of the model [2,3,7,21]. In our work, sequence of bovine serum albumin was searched by the Swiss-prot protein database provided from the Swiss Institute of Bioinformatics (ID: 2769).

The model of ketoprofen was constructed on the platform of Accelrys Discoverys Studios 2.1. The energy minimization of BSA and ketoprofen models were carried out employing CHARMm force field (Accelrys Discoverys Studios 2.1 released by the Accelrys Inc) without any constrains and minimized 2000 steps by the steepest descent method and conjugate gradient method, respectively. Ketoprofen was docked into the binding site on BSA by using Gold program (version 3.0.1, released by the Cambridge Crystallographic Data Center), a molecular docking program implemented in the Discovery Studio software suite. Gold relies on a genetic algorithm to perform conformational sampling by exploring the full range of flexible ligands and local flexible conformation of receptor to search available space for the optimal mode of interaction [6]. A scoring function, namely Goldscore, is implemented in Gold for ranking ligand binding poses [8] and obtaining the fitness value. The higher of fitness, the more stable of the complexation is. The conformation with the highest fitness was used to do further analysis. All calculations were completed on the platform of Accelrys Discoverys Studios 2.1.

The fluorescence spectra were recorded on a RF-5301PC (Shimadzu) fluorescence spectrophotometer using $150 \mathrm{~W}$ xenon lamp and $1 \mathrm{~cm}$ quartz cell. Detail procedure: firstly, shift a certain amount BSA stock solution $(5 \mu \mathrm{mol} / \mathrm{l})$ to $1 \mathrm{~cm}$ quartz cell and set the wavelength of excitation at $295 \mathrm{~nm}$, the wavelength of emission at 300-440 nm, slit widths $3.0 \mathrm{~nm} / 5.0 \mathrm{~nm}$ and scan fluorescence spectra of BSA. Secondly, add $10 \mu \mathrm{l}$ drug solution $(3 \mathrm{mmol} / \mathrm{l})$ with syringe and measure the fluorescence spectra of BSA under the same conditions and then record fluorescence intensity. Thirdly, repeat the same operation that successively add the drug solution to the quartz cell, once $10 \mu \mathrm{l}$, until the concentration of drug in the quartz cell to $0.08 \mathrm{mmol} / \mathrm{l}$. Finally, measure the fluorescence spectra of drug. The fluorescence spectra at different temperatures were measured with the same procedure to calculate the binding constants.

The UV-vis spectra were operated on a UV-1800 spectrophotometer. Detail procedure: Firstly, scan the wavelength from 200 to $400 \mathrm{~nm}$ to measure the absorption of BSA. Secondly, successively add the 
drug solution to the quartz cell, once $10 \mu \mathrm{l}$, and record the UV-vis absorption to study the interaction of BSA and ketoprofen according to the change of absorption peak.

The synchronous fluorescence spectra measurement: Measure the synchronous fluorescence spectra of BSA-ketoprofen solutions when the difference $(\Delta \lambda)$ between excitation wavelength and emission wavelength were 15 and $60 \mathrm{~nm}$, respectively. The concentration of ketoprofen increased from 0 to $0.07 \mathrm{mmol} / \mathrm{l}$.

\section{Results and discussion}

\subsection{Molecular simulation}

The sequence alignment HSA and BSA was produced using ClustalW program, which aligns multiple sequences using a progressive pairwise alignment algorithm. Based on the sequence alignment, the Accelrys Discoverys Studios 2.1 software generates a few spatial restraints from the template structure and constructed molecular models of BSA. As is shown in Fig. 2, the sequence identity of BSA and HSA is up to $75.5 \%$. Untitled1.B99990001 due to its lowest PDF total energy was chosen as the optimum conformation of BSA for further study.

The crystallographic structure of BSA obtained by homology modeling has a molecular weight of $66,430 \mathrm{~g} / \mathrm{mol}$ and contains 583 amino acids residues in a single polypeptide chain. It is known to have heart-shaped structure with a net charge of -16 on its surface and contains three homologous domains [16] and each domain can be divided into two sub-domains that A and B (Fig. 3). The two sub-domains forms a cylindrical structure and almost all the hydrophobic amino acids are embedded in the cylinder chamber to form the hydrophobic cavity [5].

The Ramachandran plot was used to assess whether the model of BSA was legitimate. It is accomplished by demonstrating the favored and disfavored conformation of protein or peptide (Fig. 4). The

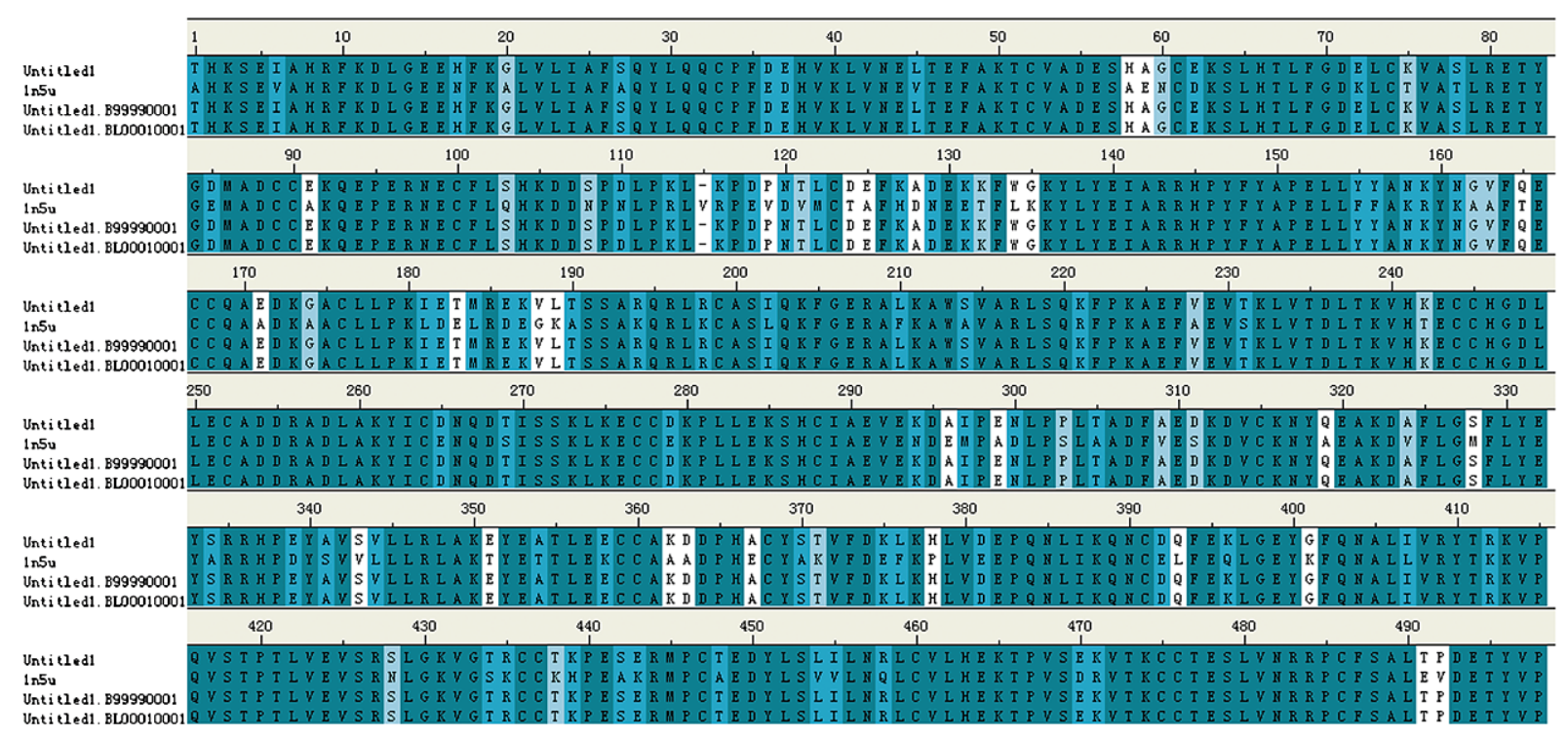

Fig. 2. Sequence alignment of BSA with template protein HAS from PDB (PDB code $\ln 5 \mathrm{u}$ ). (Colors are visible in the online version of the article; http://dx.doi.org/10.3233/SPE-2012-0549.) 


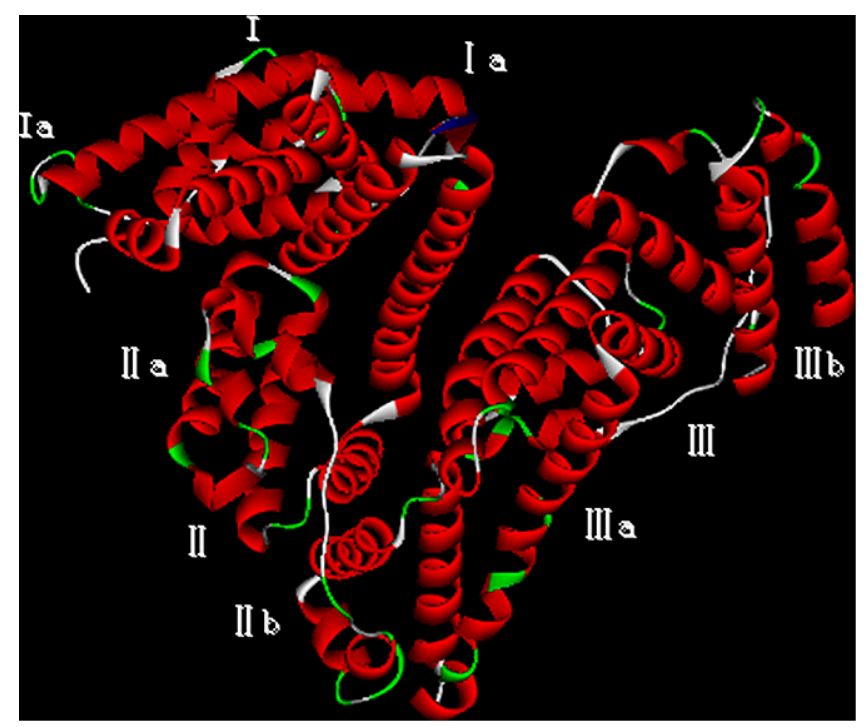

Fig. 3. The crystallographic structure of BSA. (Colors are visible in the online version of the article; http://dx.doi.org/ 10.3233/SPE-2012-0549.)

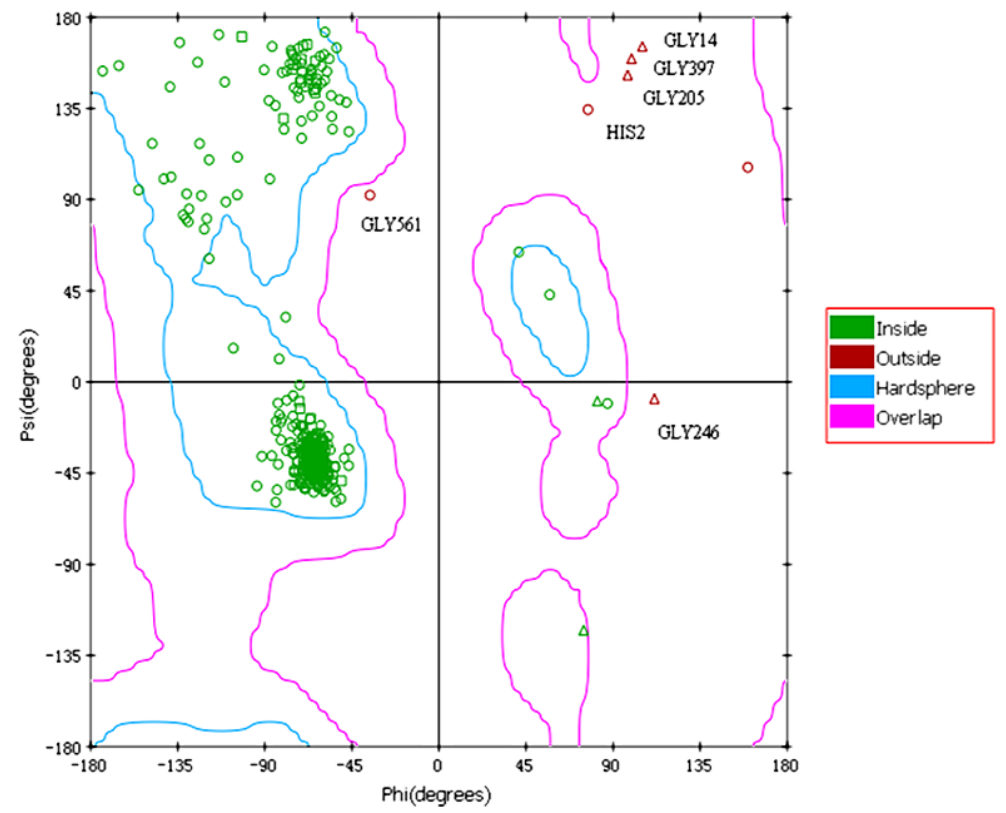

Fig. 4. Ramachandran plot obtained by evaluating the structure of BSA. (Colors are visible in the online version of the article; http://dx.doi.org/10.3233/SPE-2012-0549.)

area that amino acids often appear in could be obtained in the Ramachandran plot by analyzing known crystallographic structure. The information got from this chart would be determined whether the structural conformation of each amino acid was correct.

The blue area in Ramachandran plot was considered as "the favored region", in which the more amino acids, the more credible its structure was; purple area was considered as "the allowed area" and the points 


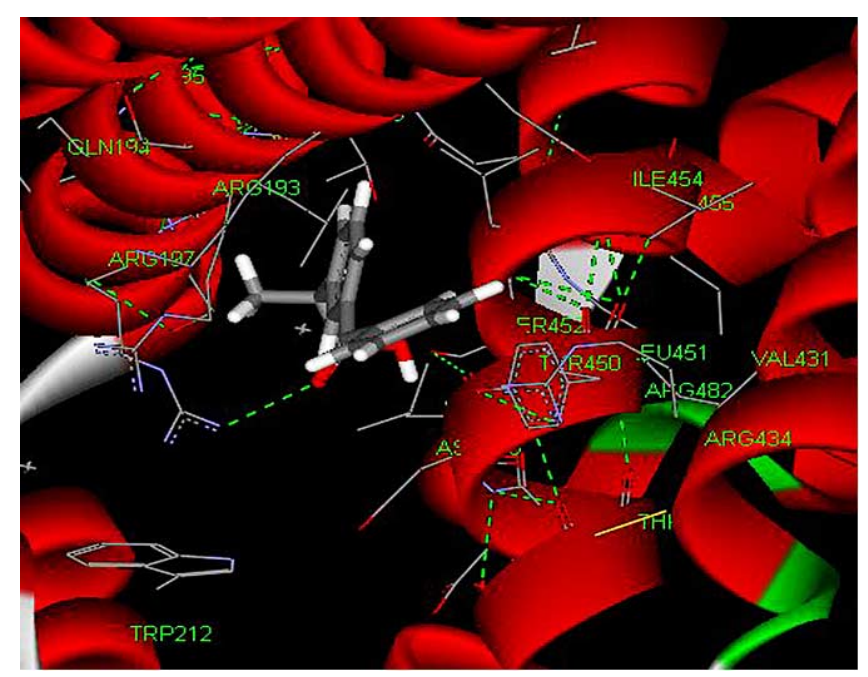

Fig. 5. Molecular docking chart of ketoprofen and BSA. Residues around the ligand $(\leqslant 1.1 \mathrm{~nm})$ were displayed. The residues of BSA were shown using line and the ligand structure was shown using stick model. Hydrogen bonds were shown using green dashed line. (Colors are visible in the online version of the article; http://dx.doi.org/10.3233/SPE-2012-0549.)

in other regions (red dots) were disallowed psi-phi amino acids conformation. As is shown in Fig. 4, more than $96 \%$ of residue $\varphi-\psi$ angles were in the favored or allowed regions, which indicated that the obtained 3D model of BSA is satisfactory. With respect to Ramachandran plot, it is observed only a few residues are in disallowed region. Residues in the unfavorable regions are far from the substrate-binding domain, which suggested these residues may not affect the ligand-protein binding simulations [18].

Molecular docking is one of the most widespread methods to investigate the protein-ligand interactions and it is an efficient technique to predict the potential ligand binding site(s) on the whole protein target. In our study, we conducted molecular docking on the basis of the active site of BSA. The conformation with the highest fitness was selected for further study.

Figure 5 displayed the molecular docking of ketoprofen and BSA and only amino acid residues within $1.1 \mathrm{~nm}$ around ketoprofen were shown. It can be seen from Fig. 5 that ketoprofen molecules in the subdomain IIA and IIIA cavity of BSA, in which there was a large hydrophobic region which can hold many drugs. A great number of green dotted lines linking amino acids represented hydrogen bonds, the presence of hydrogen bonds possibly enhanced the hydrophobicity of ketoprofen-BSA system, making ketoprofen-BSA system be a stable state. The whole molecule of ketoprofen embedded into a hydrophobic cavity formed by tryptophan (Trp212) residues, leucine (Leu196, Leu345, Leu451) residues, arginine (Arg193, Arg197, Arg482, Arg483) residues, tyrosine (Thr450) and other amino acid residues. A hydrogen bond formed between ketoprofen molecule and Arg197 residue. Thus, the bonding force to keep ketoprofen-BSA system stable was conjectured predominantly hydrophobic interaction as well as hydrogen bonds.

\subsection{Interaction of ketoprofen with $B S A$}

The UV-vis spectrum is a simple and effective method to study the interactions between drug molecules and protein. Absorption peak at $250-280 \mathrm{~nm}$ is caused by aromatic amino acids of tryptophan, tyrosine and phenylalanine. As is shown in Fig. 6, after adding ketoprofen, absorbance of BSA at maximum absorption wavelength (about $264 \mathrm{~nm}$ peaks) significantly enhanced, and the red-shifts effect 


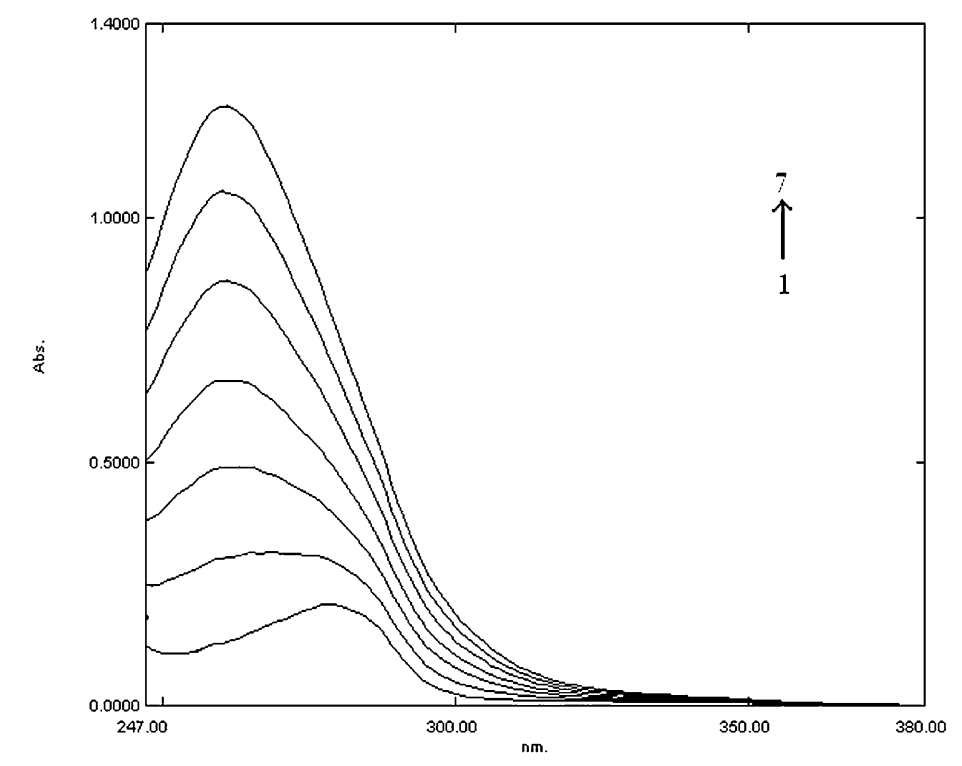

Fig. 6. UV absorption spectrum of BSA in the absence and presence of ketoprofen from 1 to 7 : $\mathrm{c}(\mathrm{BSA})=5.0 \mu \mathrm{mol} / \mathrm{l}$, c(ketoprofen)/(mmol/l): 0, 0.01, 0.02, 0.03, 0.04, 0.05, 0.06 .

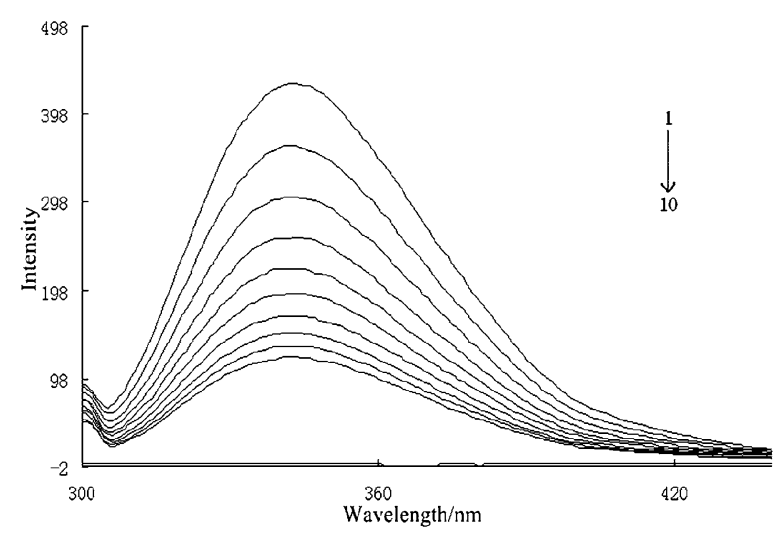

Fig. 7. The influence of ketoprofen on BSA fluorescence spectrum from 1 to $9: \mathrm{c}(\mathrm{BSA})=5.0 \mu \mathrm{mol} / \mathrm{l}, \mathrm{c}(\mathrm{ketoprofen}) /(\mathrm{mmol} / \mathrm{l})$ : $0,0.01,0.02,0.03,0.04,0.05,0.06,0.07,0.08,10, \mathrm{c}($ ketoprofen $)=3 \mathrm{mmol} / \mathrm{l}, \mathrm{c}(\mathrm{BSA})=0.0 \mu \mathrm{mol} / \mathrm{l}, \lambda_{\operatorname{exm}}=295 \mathrm{~nm}$.

can be observed at first and then blue-shift effect (278-269-264-261-261-260-261 nm). A possible reason is bovine serum albumin is a globulin, with large molecular size and loose structure, therefore, its hydrophobic cavities is often in a free state. When ketoprofen molecule was added, BSA would combine with ketoprofen to form secondary bonds, which made the complex structure more compact. Meanwhile, ketoprofen molecule inserted into the cavities of BSA and disrupted the original structure of BSA, making protein unfolding a more relaxed peptide and fluorescent amino acid residues fully exposed. This also suggested that the conformational of BSA had changed and there was a strong interaction between BSA and ketoprofen.

Figure 7 showed the fluorescence spectra of ketoprofen-BSA system in Tris-HCl buffered solution. The interaction between ketoprofen and BSA was studied by measuring the change of the intrinsic fluorescence. When the excitation wavelength was set to $295 \mathrm{~nm}$, the maximum emission wavelength 
$\left(\lambda_{\mathrm{em}}\right)$ of BSA was about $340 \mathrm{~nm}$, at this time the fluorescence adsorption of BSA mainly came from the tryptophan residue. It is shown in Fig. 7 that ketoprofen itself did not emit fluorescence; thereby, it would not interfere with the experimental results. However, as the concentrations of ketoprofen increased, the fluorescence intensity of BSA decreased regularly, which demonstrated there was strong interaction between BSA and ketoprofen. This conclusion was consistent with the result of UV-vis spectrum.

\subsection{Investigation of binding constant and binding mode}

In general, there are two types of fluorescence quenching: dynamic and static quenching. Static quenching often generates non-fluorescence substance due to mating reaction and can affect secondary structure and physiological activity of protein, while dynamic quenching is a process of energy transformation or electron transformation and do not affect conformation of protein [27]. Fluorescence intensity data were analyzed using Stern-Volmer Eq. (1) [22]:

$$
F_{0} / F=1+K_{\mathrm{q}} \tau_{0}[Q]=1+K_{\mathrm{SV}}[Q]
$$

where $F_{0}$ and $F$ are the fluorescence intensities of BSA at $340 \mathrm{~nm}$ in the absence and presence of quencher (ketoprofen), respectively; $K_{\mathrm{q}}$ is the quenching rate constant of the biomolecule; $[Q]$ is the concentration of quencher; $\tau_{0}$ is the average fluorescence life time of biomolecule without quencher; $K_{\mathrm{SV}}$ is the Stern-Volmer fluorescence quenching constant. The larger the value of $K_{\mathrm{SV}}$, the stronger interaction between BSA and ketoprofen will be.

The fluorescence spectra of BSA and ketoprofen were determined on 298,310 and $318 \mathrm{~K}$ at pH 7.40 Tris- $\mathrm{HCl}$ buffered solution to investigate the fluorescence quenching mechanism. Figure 8 was obtained by analyzing fluorescence spectra data using Stern-Volmer Eq. (1). $K_{\mathrm{SV}}$ was calculated and listed in Table 1; all of the linear correlation coefficients were more than 0.99 . The corresponding quenching constants were obtained from $K_{\mathrm{SV}}=K_{\mathrm{q}} \tau_{0}$. Since the maximum quenching constant of dynamic fluorescence is $0.2 \mathrm{mmol} / 1$ [1], $K_{\mathrm{SV}}$ in our study is larger than this value. Judge by this the quenching process was caused by static quenching. Table 1 also showed that with temperature going up, $K_{\mathrm{SV}}$ increased, which indicated that the quenching process was caused by dynamic quenching. Thus, fluorescence quenching of BSA caused by ketoprofen was not only a single one but simultaneously static and dynamic quenching.

The acting forces between small molecule drugs and biomolecule mainly include van der Waals' force, hydrogen bond, electrostatic force and hydrophobic force. Different drugs may have different types of binding force when interaction with protein. However, binding types can be roughly determined

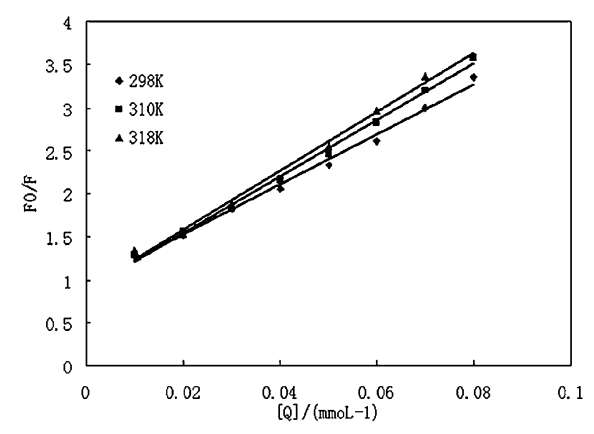

Fig. 8. Stern-Volmer plots for BSA fluorescence quenching by different ketoprofen. $\mathrm{c}(\mathrm{BSA})=5.0 \mu \mathrm{mol} / \mathrm{l}$. 
Table 1

Stern-Volmer quenching constants and the correlation coefficients for the system of ketoprofen-BSA at 298, 310 and $318 \mathrm{~K}$

\begin{tabular}{lcccccc}
\hline$T$ & Liner equation & $R^{2}$ & $\begin{array}{c}K_{\mathrm{SV}} \\
\left(10^{3} 1 / \mathrm{mol}\right)\end{array}$ & $\begin{array}{c}\Delta G^{\Phi} \\
(\mathrm{KJ} / \mathrm{mol})\end{array}$ & $\begin{array}{c}\Delta H^{\Phi} \\
(\mathrm{KJ} / \mathrm{mol})\end{array}$ & $\begin{array}{c}\Delta S^{\Phi} \\
\left(\mathrm{J} \cdot \mathrm{mol}^{-1} \cdot \mathrm{K}^{-1}\right)\end{array}$ \\
\hline 298 & $y=29.033 x+0.9395$ & 0.9929 & 29.033 & -25.01 & 74.514 & 333.98 \\
310 & $y=32.892 x+0.8822$ & 0.9957 & 32.892 & -29.02 & & \\
318 & $y=34.51 x+0.8768$ & 0.9904 & 34.51 & -31.69 & & \\
\hline
\end{tabular}

by the thermodynamic parameters of interaction. When the temperature does not vary significantly, the reaction enthalpy can be considered as a constant. According to Van't Hoff's law and Gibbs free energy equations:

$$
\begin{aligned}
& \ln K=-\Delta H^{\Phi} / R T+\Delta S^{\Phi} / R, \\
& \Delta G^{\Phi}=\Delta H^{\Phi}-T \Delta S^{\Phi} .
\end{aligned}
$$

Thermodynamic parameters of BSA and ketoprofen at different temperature were obtained and listed in Table 1. It was mentioned above that the thermodynamic parameters $(\Delta H, \Delta S)$ before and after reaction can be used to determine the type of interaction: when $\Delta H>0, \Delta S>0$, the acting force was hydrophobic force; when $\Delta H<0, \Delta S<0$ it was van der Waals' force and hydrogen bond; and when $\Delta H<0, \Delta S>0$, it was electrostatic force [17]. It was displayed in Table 1 that the fact $\Delta G^{\Phi}<0$ proved that the reaction was spontaneous, and that $\Delta H>0, \Delta S>0$ proved the acting force type was hydrophobic force.

\subsection{Determination of qualitative and quantitative basis about the influence of drugs to protein secondary structure}

The measurements of synchronous fluorescence can provide some important information about the microenvironment of protein. In this work, the synchronous fluorescence spectroscopy was used to further investigate the conformational changes of BSA in the presence of ketoprofen. Synchronous fluorescence spectra of tryptophan residue and tyrosine residue were obtained at $\Delta \lambda=60$ and $\Delta \lambda=15 \mathrm{~nm}$, respectively. Figure 9 showed when fixed concentration of protein, with concentrations of ketoprofen increasing, the maximum emission wavelength of synchronous fluorescence spectra unchanged when $\Delta \lambda=15 \mathrm{~nm}$, while when $\Delta \lambda=60 \mathrm{~nm}$, synchronous fluorescence spectra moved from 343 to $347 \mathrm{~nm}$. The maximum emission wavelengths of tryptophan residue and tyrosine residue in the protein molecule are related to the polarity of their surroundings, thus, changes of the maximum emission wavelengths will represent conformational change of protein [28]. That the maximum emission wavelength amino acids residues had a blue shift indicated the decreased hydrophilicity of chromophore microenvironment, structure of protein became closer and the red shift suggested that hydrophobicity of chromophore microenvironment decreased, structure of protein became loose [26]. Therefore, the addition of ketoprofen changed the microenvironment of BSA tryptophan residues, making polarity of BSA hydrophobic cavity increase and hydrophobicity decrease, meanwhile, internal hydrophobic structure of BSA had a little collapse, stretching degree of peptide chain increased.

\subsection{Energy transfer mechanisms and binding distance between ketoprofen and BSA}

Dipole-dipole non-radiative Forster energy transfer theory was used as determining the distances between the BSA residue and ketoprofen bound to BSA. According to this theory, the energy transfer effect 


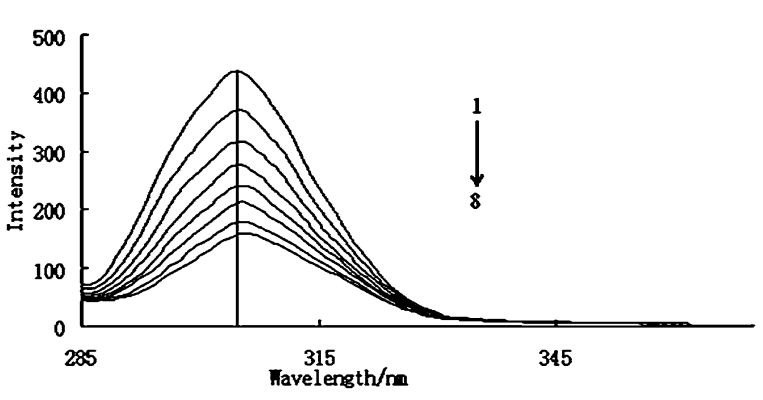

(a)

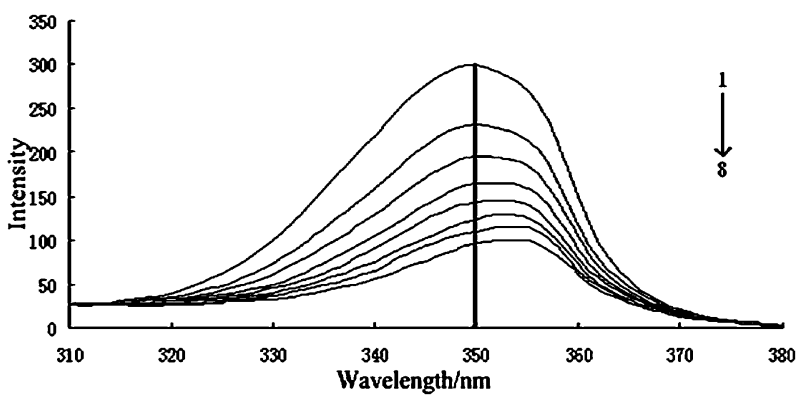

(b)

Fig. 9. Effect of ketoprofen on the synchronous fluorescence spectra of BSA $\Delta \lambda=15 \mathrm{~nm}$ (a) and $\Delta \lambda=60 \mathrm{~nm}$ (b).

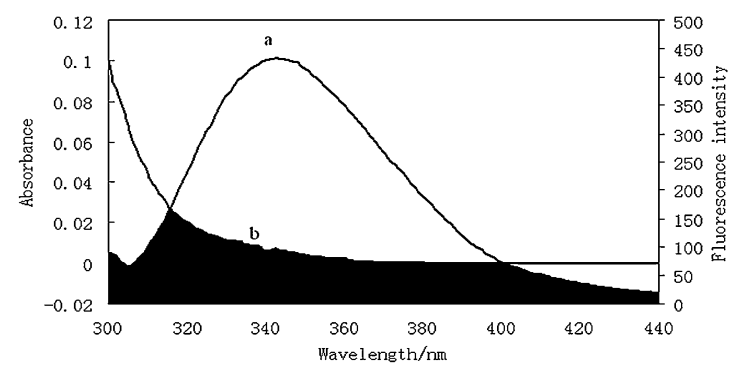

Fig. 10. The fluorescence emission spectrum of BSA (a) and the absorption spectrum of ketoprofen (b).

is not only related to the distance between the donor and acceptor, but influenced by the critical energy transfer distance $R_{0}$ :

$$
\begin{aligned}
& E=\frac{R_{0}^{6}}{R_{0}^{6}+r^{6}}=\frac{F_{0}-F}{F_{0}}, \\
& R_{0}^{6}=8.8 \times 10^{-25} K^{2} n^{-4} \varphi J, \\
& J=\frac{\sum F(\lambda) \varepsilon(\lambda) \lambda^{4} \Delta \lambda}{\sum F(\lambda) \Delta \lambda},
\end{aligned}
$$

where $E$ is the efficiency of energy transfer, $R_{0}$ is the critical energy transfer distance when the efficiency of energy transfer $E$ is $50 \%, r$ is the distance between the donor and the acceptor, $K^{2}$ is the spatial orientation factor of the dipole, $n$ is the refractive index of the medium, $\varphi$ is the fluorescence quantum yield of the donor, $J$ is the over lapped integral of the fluorescence emission spectrum of donor and the absorption spectrum of acceptor. $F(\lambda)$ is the fluorescence intensity of the fluorescent donor and $\varepsilon(\lambda)$ is the molar absorbance coefficient of the acceptor at wavelength $\lambda$.

Figure 10 displayed when molar ratio is 1:1, the spectral overlap of UV absorption spectrum of ketoprofen and fluorescence emission spectrum of BSA. Under experimental conditions, often $K^{2}=2 / 3$, $\varphi=0.15, n=1.336$, therefore, $E, J, R_{0}$ and $r$ were calculated to be $0.11,1.12 \times 10^{-17} \mathrm{~cm}^{3} \cdot 1 / \mathrm{mol}$, $1.58 \mathrm{~nm}$, respectively according to Eqs (4)-(6), which was accord with non-radiative energy transfer theory (BSA and ketoprofen were close enough $(r<8 \mathrm{~nm})$ ).

The quenching mode of NASID drugs to BSA (e.g., phenylbutazone, ibuprofen) [20] was found to be a combination of static and dynamic quenching, the binding force was hydrophobic force, and the distance 
between NASID drugs (phenylbutazone, ibuprofen) and BSA residues were 1.57, $1.54 \mathrm{~nm}$, respectively. In our study, the information about interaction of ketoprofen and BSA was similar to above. Therefore, we can conclude that there are some commonness on interaction between the NASID drugs and BSA.

\section{Conclusion}

In this work, the interaction between ketoprofen and BSA was investigated by molecular simulation method in combination with fluorescence, UV-vis absorption spectra under the physiological conditions. The experimental results showed that ketoprofen had strong ability to quench the intrinsic fluorescence of BSA through a static coupled with dynamic quenching mechanism; the binding constant was $K_{\mathrm{A}}=2.90 \times 10^{4} \mathrm{l} / \mathrm{mol}(298 \mathrm{~K})$ and binding distance $r=1.58 \mathrm{~nm}$. Information obtained from UV-vis absorption spectrum and synchronous fluorescence suggested that the conformation of BSA changed when combining with ketoprofen. The results of thermodynamic parameters obtained indicated that hydrophobic force and hydrogen bond were predominant forces to keep stable the complex. Moreover, molecular simulation studies revealed that the ketoprofen was in the sub-domain IIA and IIIA of the BSA.

\section{Acknowledgements}

The work was supported by National Found for Fostering Talents of Basic Science (NFFTBS). We also thank Lun Li, Jia He, Xue Lian Jia, Wan Yin Yang and Wei Wei Fang for supporting our work greatly.

\section{References}

[1] Y. Cui and J.Q. Chen, Chinese J. Spectroscopy Lab. 27 (2010), 1064-1069.

[2] L.R. Forrest, C.L. Tang and B. Honig, Biophys. J. 91 (2006), 508-517.

[3] K. Ginalski, Curr. Opin. Struct. Biol. 16 (2006), 172-177.

[4] W.Y. He, G.Y. Chen and J. Du, Acta Chim. Sinica 66 (2008), 2365-2370.

[5] Z.Q. Jiang, Y.H. Chi, J. Zhuang et al., Spectrosc. Spect. Anal. 27 (2007), 986-990.

[6] G. Jones, P. Willett and R.C. Glen, J. Mol. Biol. 245 (1995), 43-53.

[7] M.Y. Li and B.H. Wang, J. Mol. Model. 13 (2007), 1237-1244.

[8] Y. Li, B.C. Zhou and R.X. Wang, J. Mol. Graphics Modell. 28 (2009), 203-219.

[9] B. Liu and Y. Guo, J. Lumin. 130 (2010), 1036-1043.

[10] F.F. Liu and X.Y. Dong, J. Chromatogr. A 1175 (2007), 249-258.

[11] F.F. Liu, X.Y. Dong, T. Wang and Y. Sun, J. Chromatogr. A 1175 (2007), 249-258.

[12] J.Q. Liu and J.N. Tian, Pharm. Biomed. Anal. 35 (2004), 671-674.

[13] Y.M. Liu, G.Z. Li and W.K. Song, Acta Phys. Chim. Sin. 12 (2006), 1456-1459.

[14] S.Y. Lu, Y.J. Jiang, J. Lv and T.X. Wu, J. Mol. Graphics. Modell. 28 (2010), 766-774.

[15] A.L. Ong and A.H. Kamaruddin, Process Biochem. 40 (2005), 3526-3535.

[16] W. Qi and Z.M. He, Comput. Appl. Chem. 23 (2006), 821-823.

[17] P.D. Ross and S. Sabramanian, Biochem. 20 (1981), 3096-3401.

[18] M. Shahlaei, A. Madadkar Sobhani, K. Mahname, A. Fassihi, L. Saghaie and M. Mansourian, Biochim. Biophys. Acta 1808 (2011), 802-817.

[19] J. Sponer and N. Spackova, Methods 43 (2007), 278-290.

[20] Y.T. Sun, Y.P. Zhang, Chem. J. Chinese University 30 (2009), 1095-1100.

[21] A. Tramontano, Meth. Companion Meth. Enzymol. 14 (1998), 293-300.

[22] C.X. Wang, F.F. Yan, Y.X. Zhang and L. Ye, Photochem. Photobiol. A 192 (2007), 23-28. 
[23] G.K. Wang, C.L. Yan and X.M. Lu, Acta Chim. Sinica 67 (2009), 1967-1972.

[24] J. Wang, Y.W. Guo and B. Liu, J. Lumin. 131 (2011), 231-237.

[25] J.F. Xiao, Z.R. Guo, Y.S. Guo, F.M. Chu and P.Y. Sun, J. Mol. Graphics Modell. 25 (2006), 289-295.

[26] C.N. Yan, H.X. Zhang, Y. Liu et al., Acta Chim. Sinica 63 (2005), 1727-1732.

[27] J.H. Yu, B. Li and P. Dai, Spectrochim. Acta, Part A 74 (2009), 277-281.

[28] H.J. Zhang and H. He, Acta Chim. Sinica 17 (2010), 1741-1748. 


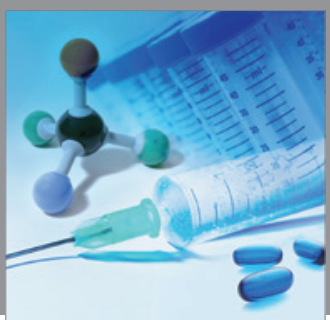

International Journal of

Medicinal Chemistry

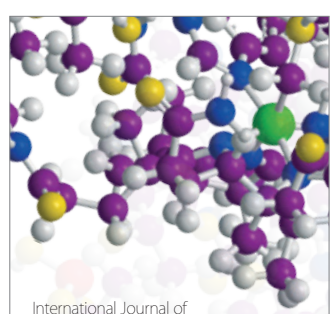

Carbohydrate Chemistry

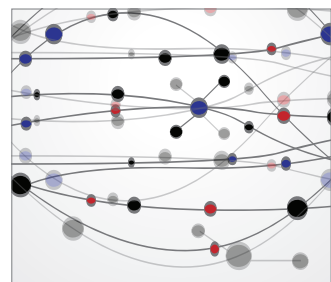

The Scientific World Journal
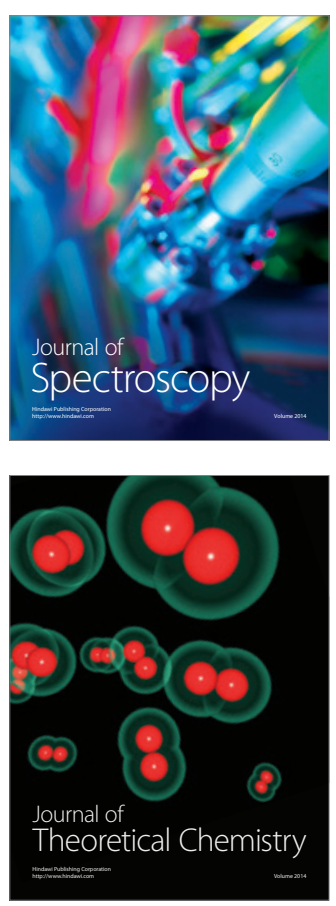
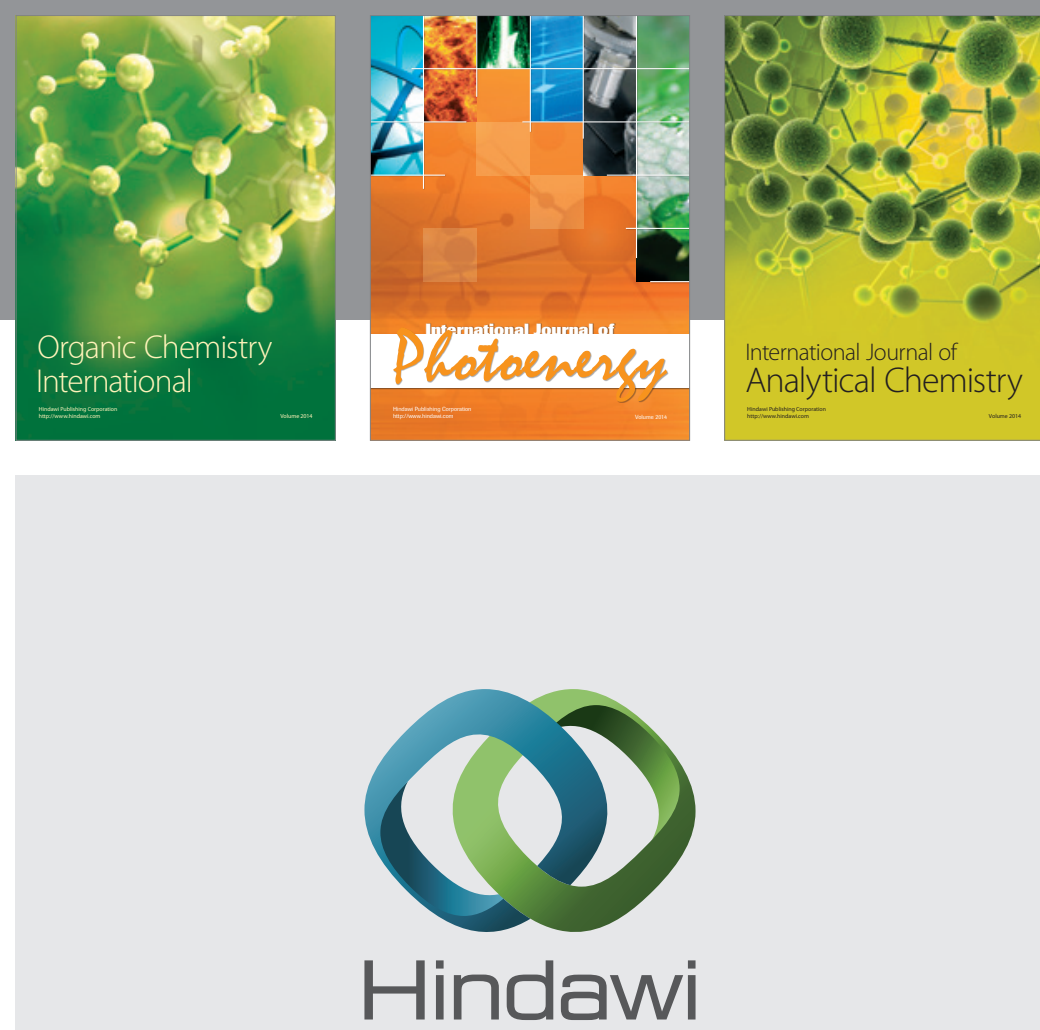

Submit your manuscripts at

http://www.hindawi.com
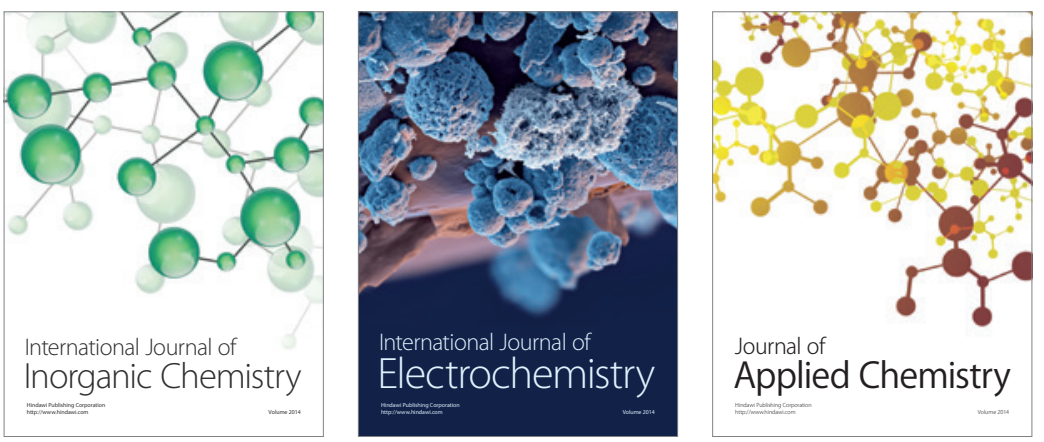

Journal of

Applied Chemistry
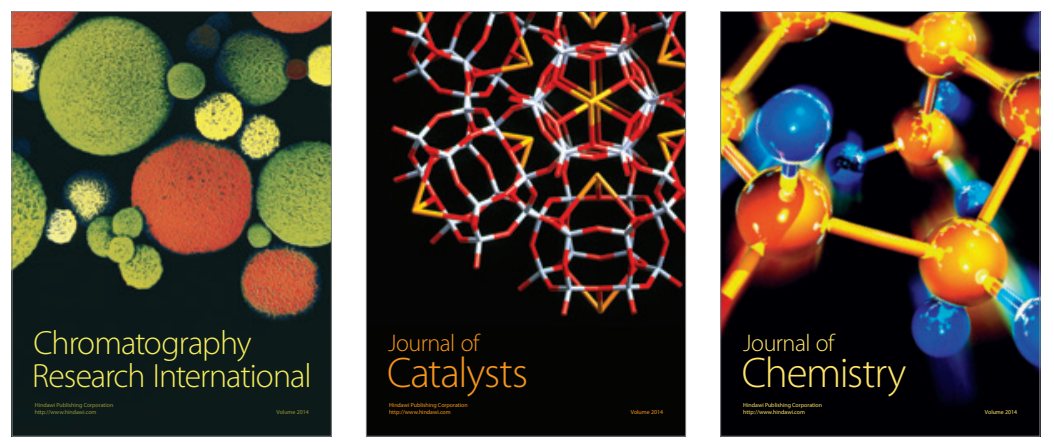
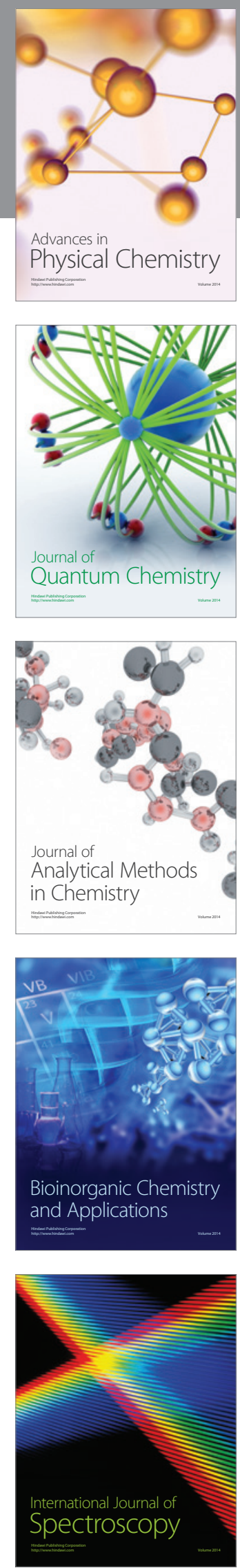